\title{
Mobile Phone Background Design for Older Adults: A Case Study of Line
}

\author{
Kleddao Satcharoen
}

\begin{abstract}
The purpose of this study was to examine how visual characteristics like the background image could affect usability of mobile apps for older adults, using a case study of the Line messaging application. Older adults typically have a lower use of mobile Internet technologies, because of both visual and cognitive barriers and attitudes and resistance to change. The study was conducted as a qualitative observational study of 40 older adults, who were offered a brief training session on Line and then monitored at three times over a three-week period. The findings showed that post-training, many participants did change their backgrounds to suit colour preferences, mood, or vision needs. Adopters also self-discovered features like group-based background colours. Although there were a few non-adopters remaining, the study showed that training in app use encouraged senior users to use the app and to adapt it to their needs. The implication is that app-specific training could improve senior use of mobile apps.
\end{abstract}

Index Terms-Background design, line application, older adults, color.

\section{INTRODUCTION}

Although use of the Internet and mobile Internet access is increasingly common around the world, it is not yet ubiquitous. One notable gap in access that remains in the so-called digital divide is access by the elderly [1]. The Internet and mobile computing offers older adults tools that could substantially improve their living conditions, such as access to online shopping, inexpensive communication tools to keep in touch with family and friends, and access to information about medical, financial, social, and other requirements. Despite this, as [1] explain, many older adults resist the adoption of information technologies, either due to lack of interest or due to barriers like lack of knowledge about technology, the cost of adoption, or physical and cognitive barriers.

Mobile phones offer even more potential advantages than fixed computing services, since they are relatively inexpensive, easy to use, and portable [2]. Despite this, [2] found that nearly half of elderly users surveyed had negative social and instrumental attitudes about the use of mobile phones. Visual limitations and visual confusion can be a significant barrier for older adults in learning to use mobile phones and other mobile computing devices [3].

The purpose of this study is to examine how visual characteristics, such as the background image, can improve usability of mobile apps for older adults. To do so, a case

Manuscript received January 9, 2019; revised March 7, 2019.

Kleddao Satcharoen is with King Mongkut's Institute of Technology Ladkrabang, Bangkok, Thailand (e-mail: kleddao@gmail.com). study of the Line application was conducted.

\section{LINE}

Line is a leading multi-channel mobile communications app that allows users to communicate using either mobile or Wi-Fi access. Line allows users to communicate in different ways, including voice over IP (VoIP), text-based messaging and texts, and exchange of multimedia messages. Owned by NHN Corporation, a South Korean company active in web search and communications, Line was released following the 2011 Japanese earthquake and tsunami, which revealed weaknesses in Japan's communication networks [4]. The communication model of allowing consumers to use mobile minutes or other communication approaches was revolutionary at the time [4], although it is now used by several leading communication models.

Line had an estimated 203 million monthly active users as of January 2018 [5]. Although it is available globally, Line is most popular in Asian countries. Thailand was one of Line's first international markets. As of 2014, Thailand had an estimated 33 million registered users [6]. Line is now one of the most popular apps in Thailand, with an estimated $93 \%$ of mobile computing users being registered for the app [7]. This makes Line the most popular app among Thai smartphone users, even ahead of Facebook (used by about $80 \%$ of connected users) [7].

\section{Factors In Use of Mobile Phones by OldER AdUlts}

Factors related to mobile phones by older adults include perceptual and cognitive issues and attitudes and behaviours.

\section{A. Perceptual and Cognitive Issues}

Visual limitations and vision loss are associated with aging, with one study reporting that 1 in 28 people over age 40 are classified as low vision [8]. The rate of vision loss accelerates with age, with many older adults having poor enough vision that they may struggle with daily functional activities [8]. Furthermore, there are vision problems such as glaucoma and cataracts that primarily affect older adults [9]. Visual limitations can be a significant barrier for older adults using mobile computing devices, especially when it is not possible to adapt the visual interface (for example increasing font size or changing display colour) [3]. Age-related vision changes affect colour preferences because of changes in cone-contrast channels [10]. They found that elderly subjects were more likely to prefer red and green hues than blue hues compared to younger participants, who generally preferred blue hues [10]. This was consistent with previous studies they reviewed. The 
elderly may also struggle with perception of complex images if they have working memory deficits [11]. Finally, age-related cognitive barriers can make mobile phones and other technologies difficult to learn to use [12].

\section{B. Attitudes and Behaviors}

While older adults do use mobile phones, they often have negative attitudes surrounding their utility and social status. For example, one study showed that around $45 \%$ of female seniors and $41 \%$ of male seniors had negative attitudes about the utility or sociocultural meaning of mobile phones [2]. An IS adoption perspective on mobile phone use also showed that senior users may be reluctant to adopt the technology because they do not view them as useful [1]. Older users may have poor technology skills, which negatively affect how they view the technology [12]. Seniors may view mobile phones as difficult to learn or unnecessary [12]. This can cause anxiety, which negatively affects mobile phone use [13]. At the same time, users that are equipped with appropriate knowledge and have intrinsic motivations and use expectations will adopt the technology [13].

\section{BACKGROUND DESIGN}

The background of a computer display or app is the static ground on which active components such as icons, menus, or links are displayed [14]. According to Gestalt theory, the visual field is divided into the figure (which is the visual focus of the scene) and the ground (which is everything else in the visual field) [15]. The ground, or background, is not typically a field of interaction for the viewer, but the contrast between the figure and ground can still affect perception and response to the figure [15]. Thus, the background is an important aspect of the interaction field between the user and the computer, though it is not typically the focus of design [14].

Despite the potential importance of the visual background and background design in the user experience of an app, there has been little attention paid to how users respond to background characteristics. Studies have shown that manipulation of background characteristics to emphasize or hide specific aspects of an image can provoke cognitive responses such as fear, although more extensive research is required to fully understand this response [16]. Research that was focused on notice and recall of app warnings showed that up to $80 \%$ of users could recall the app background colour, a far higher rate than recalled the app warning [17].

The choice of background image is particularly important for elderly users. A technical report has demonstrated that choice of background images, particularly rich background images, is a usability challenge for elderly web users [18]. Furthermore, only $24 \%$ of elderly mobile users in one study ever changed their background image [19]. Thus, selecting the right background image the first time will have a significant effect on app usability for older adults.

\section{Methodology}

The study used an observational qualitative design, with open-ended descriptive analysis used to analyse the findings
[20]. 40 older adults (aged 60 and above) were recruited to participate in the study. Participants were already mobile phone users, but did not necessarily have the Line program installed or use it regularly. Recruitment took place through neighbourhood announcements and announcements in local drop-in centres and other congregation areas for older adults. Participants were asked to telephone or email the researcher, to avoid participant bias resulting from existing familiarity with technology.

The research was a longitudinal study, with repeated observations taking place over a period of three weeks. The research began with an introduction to the study and a 20-minute training session, in which respondents were taught to change the background in the Line application. These training sessions took place in groups of 10, with training sessions staggered a few days apart to provide participants with flexibility about the timing of their session.

Three follow-up contacts were conducted with study participants in the initial study groups. These follow-ups took place at 7, 14, and 21 days after the initial training session for each participant group. Follow-up contacts addressed use of the Line application and the user behaviour related to changing the background of the Line application. Respondents were asked about whether they were using Line and whether that had changed from the previous period; whether they had changed the background (and if so, details of the change); and why they had changed it. Researchers also checked the Line application to determine whether changes had been made. The results were collated and examined to understand whether participants were willing to change their use of Line following training.

\section{RESULTS}

\section{A. Initial Line Use}

At the initial sessions, the researcher ensured all participants had Line installed and spent 20 minutes showing participants how to use Line and change the background, but did not offer any guidance on choice of background. At this session, 36 out of $40(90 \%)$ had the Line application installed on their mobile phones. However, only 26 participants $(65 \%)$ were active users (within one month). Five participants (12.5\%) had bought the phone with the Line app installed but never used it. The remainder (five participants or 12.5\%) had used it at one time, but stopped. Six participants (15\%) had something other than the default background. Of these, four participants $(10 \%)$ had someone else, such as a child or niece or nephew, change it for them. Only two participants $(5 \%)$ had changed it themselves. Both were daily Line users.

\section{B. Seven-Day Follow-up}

At the initial sessions, the researcher ensured all participants had Line installed and spent 20 minutes showing participants how to use Line and change the background, but did not offer any guidance on choice of background. At this session, 36 out of $40(90 \%)$ had the Line application installed on their mobile phones. However, only 26 participants $(65 \%)$ were active users (within one month). Five participants (12.5\%) had bought the phone with the Line app installed but 
never used it. The remainder (five participants or $12.5 \%$ ) had used it at one time, but stopped. Six participants (15\%) had something other than the default background. Of these, four participants (10\%) had someone else, such as a child or niece or nephew, change it for them. Only two participants (5\%) had changed it themselves. Both were daily Line users.

\section{Fourteen-Day Follow-up}

37 participants $(92.5 \%)$ used Line within the past seven days, and the remaining three participants $(7.5 \%)$ had not. Six participants $(15 \%)$ still did not change their background colour, while the remainder changed it again. Five participants (12.5\%) changed their standard chat background to a vivid colour. 26 participants $(65 \%)$ changed their backgrounds to a pastel colour. Furthermore, three participants $(7.5 \%)$ changed their backgrounds to multiple colours to identify caller groups (friends and family, etc.) or for specific callers. Many participants stated they changed the colour again because of boredom.

\section{Twenty-One-Day Follow-up}

Usage of the Line app was unchanged from the previous session. Colour preferences also continued, with 26 participants $(65 \%)$ changing to a pastel hue; five participants $(12.5 \%)$ changing to a vivid colour; and six participants $(15 \%)$ not changing their backgrounds. Nine participants $(22.5 \%)$ chose a different colour from the previous week. Eight participants (20\%) used multiple backgrounds for caller groups this week. The new adopters of this feature said they had gotten the idea by experimenting during a chat. While users who had chosen pastel colours did not state a reason for the preference, the few participants that preferred a brighter hue identified two main reasons. One reason expressed by three people was that they spent a lot of time in hospitals and other areas where it could be difficult to see, and the bright hue helped with reading. Another reason, also expressed by three people, was that the brighter hues cheered them up. Respondents who did not change their backgrounds during the three weeks were asked why they had not in this session. The most common reason was that they rarely used Line (two or three times a week).

\section{SUMMARY}

Training in how to change background colours resulted in a rapid increase in the behaviour. From only two participants in the training session, at the end of the session 31 participants were changing their backgrounds. Participants engaged in self-discovery of Line features, customizing their background colours to caller groups. The rate of Line use also grew, going from 26 regular users to 37 regular users. The choice of background colours showed a clear preference for pastel colours, but also a group that strongly preferred vivid colours for reasons including mood and lighting in their environment. There were three participants that had not used Line by the end of the session.

\section{DISCUSSION}

The study was consistent with existing research on attitudes and beliefs about technology use. For example, consistent with [2], the study did show that some users had negative beliefs about the Line app. These beliefs included lack of belief about the usefulness of the app and negative self-efficacy beliefs (such as not thinking they could change the background on their own). It is also notable that the resistant users, who did not make use of the Line app by the end of the study, also showed negative beliefs about the utility of the study, consistent with another previous study of senior IS use [1]. For example, one non-user throughout the study stated that he had no one to talk to on the service. Thus, the attitudes and beliefs about the technology were consistent with what was expected. What was not expected was that a brief training session and transfer of information about how to use the app could increase usage so much. This may be because it improved technology skills and reduced anxiety, which are factors known to affect willingness to use technology [12], [13]. The finding that at least some users, once given initial information about how to change backgrounds, were able to discover more nuanced details (for example, how to assign colours to user groups) also supports the importance of reducing anxiety and improving technology skills. Thus, this research does provide support for the idea that improving training and information for seniors to improve their understanding of technology can also improve their willingness to try and use the technology.

The reasons for choosing particular backgrounds found in this study are novel. The previous research has not routinely addressed background image or colour use, which is a significant gap in the literature. This study did show that users tended to choose solid colours, rather than images, for their backgrounds. This could be because of the problem of visual processing with complex images [11], although it could also be because the initial training session used a solid colour background. The study showed distinct colour preference groupings, with about five times as many users choosing pastel colours rather than vivid colours. Users who chose pastels appeared to be driven by simple colour preference. However, those who chose the vivid colours had specific cognitive or affective reasons underlying these choices, including that they were easier to see and improved mood. The visual benefit of vivid colours could be related to vision impairments, which are a well-known factor in the usability of mobile computing devices [8], [3]. By making the device easier to see in a common environment, the background change to a brighter colour has improved device usability. What was not expected was that some users might choose bright colours to influence their mood - a possibility completely unexplored in previous studies.

\section{CONCLUSION}

This study has explored the effect that direct tuition on the background of a common mobile app (Line) could improve usability and adoption for older adult users. Using a brief training session, followed by a three-week observational period, the study revealed several key issues. First, user adoption of Line could be improved by even a brief tutorial on how to use the tool. This extended to ability and willingness to 
change the app background, which few users had done previously. Second, once taught how to use the app and change the background, users were more likely to do so to meet both their colour preferences and their functional needs. At least some users discovered some features of the app themselves, such as group-based background colour coding. Others chose to adapt their background use to meet needs such as improving visibility and mood. This study was an exploratory and observational study, which does mean that there are some significant limitations including focus in one geographic area and age group. However, it does provide evidence for the importance of the background in app design and offers opportunities for further research. One of the most promising areas is the potential influence of app backgrounds on mood, following the evidence from a small number of participants who stated that they chose bright colours to positively influence their mood. Although there is some limited evidence about the importance of colour in mood, none of the previous research has extended to app backgrounds. This is part of a general dearth of research on the topic of computing backgrounds. However, it is potentially important especially as people spend more and more time interacting with mobile computing. Thus, examining this potential connection is the main recommendation for further study.

\section{ACKNOWLEDGMENT}

I would like to thanks the participants for participating in this study. I appreciate your honesty and willingness to assist with this important research.

\section{REFERENCES}

[1] B. Niehaves and R. Plattfaut, "Internet adoption by the elderly: Employing IS technology acceptance theories for understanding the age-related digital divide," European Journal of Information Systems, vol. 23, no. 6, pp. 708-726, 2014.

[2] N. Navabi, F. Ghaffari, and Z. Jannat-Alipoor, "Older adults' attitudes and barriers toward the use of mobile phones," Clinical Interventions in Aging, vol. 11, pp. 1371-1378, 2016.

[3] H. S. Tsai, R. Shillair, and S. R. Cotten, "Social support and 'playing around': An examination of how older adults acquire digital literacy with tablet computers," Journal of Applied Gerontology, vol. 36, no. 1, pp. 29-55, 2015.

[4] M. Saito. (2012). Born from Japan disasters, line app sets sights on U.S., China. [Online]. Available: https://www.reuters.com/article/japan-app-line/born-from-japan-disas ters-line-app-sets-sights-on-u-s-china-idUSL2E8JD0PZ20120816

[5] Statista. (2018). Most popular global mobile messenger apps as of January 2018, based on number of monthly active users (in millions). [Online]. Available: https://www.statista.com/statistics/258749/most-popular-global-mobi le-messenger-apps/

[6] Statista. (2018). Number of registered LINE app users in selected countries as of October 2014 (in millions). [Online]. Available: https://www.statista.com/statistics/250927/number-of-registered-lineapp-users-in-selected-countries/

[7] Z. Lawrence. (2017). LINE app dominates in Thailand. [Online]. Available: https://sea.kantar.com/tech/social/2017/line-app-dominates-in-thailan d/

[8] S. Berger and F. Porrell, "The association between low vision and function," Journal of Aging and Health, vol. 20, no. 5, pp. 504-525, 2018.

[9] P. Y. Ramulu, S. W. V. Landingham, R. W. Massof, E. S. Chan, L. Ferruci, and D. S. Friedman, "Fear of falling and visual field loss from glaucoma," Opthalmology, vol. 119, no. 7, pp. 1352-1358, 2012.

[10] Y. Ling and A. Hurlbert, "Age-dependence of colour preference in the UK population," in New Directions in Colour Studies, C. P. Biggam, C. A. Hough, C. J. Kay, and D. R. Simmons, Eds.. Amsterdam: John Benjamins Publishing Company, 2011, pp. 347-360.

[11] A. Sherman, M. Grabowecky, and S. Suzuki, "In the working memory of the beholder: Art appreciation is enhanced when visual complexity is compatible with working memory," Journal of Experimental Psychology: Human Perception and Performance, vol. 41, no. 4, pp 898-903, 2015.

[12] N. Charness and W. R. Boot, "Aging and information technology use," Current Directions in Psychological Science, vol. 18, no. 5, pp. 253-258, 2009.

[13] K. H. Wang, G. Chen, and H. G. Chen, "A model of technology adoption by older adults," Social Behavior and Personality: An International Journal, vol. 45, no. 4, pp. 563-572, 2017.

[14] J. Preece, H. Sharp, and Y. Rogers, Interaction Design: Beyond Human-Computer Interaction, 4th ed. Hoboken, NJ: John Wiley and Sons, 2015.

[15] J. Wagemans, "Historic and conceptual background: Gestalt theory," Oxford Handbook of Perceptual Organization, Oxford: Oxford University Press, 2015

[16] S. Maren, K. L. Phan, and I. Liberzon, "The contextual brain: Implications for fear conditioning, extinction and psychopathology," Nature Reviews Neuroscience, vol. 14, pp. 417-428, 2013.

[17] R. Balebako, F. Schaub, I. Adjerid, A. Acquisti, and L. F. Cranor, "The impact of timing on the salience of smartphone app privacy notices," in Proc. the 5th Annual ACM CCS Workshop on Security and Privacy in Smartphones and Mobile Devices, 2015, pp. 63-74

[18] D. Lunn, Y. Yesilada, and S. Harper. (June, 2009) Barriers faced by older users of static web pages: Criteria used in the barrier walkthrough method (HCW - SCWeb2 Technical Report WP1 D1 and RIAM Technical Report Deliverable 3.1). [Online]. Available: http://citeseerx.ist.psu.edu/viewdoc/download?doi=10.1.1.613.7905\& rep $=$ rep $1 \&$ type $=$ pdf

[19] J. Balata, Z. Mikovec, and T. Slavicek, "KoalaPhone: Touchscreen mobile phone UI for active seniors," Journal of Multimodal User Interfaces, vol. 9, no. 4, pp. 263-273, 2015

[20] S. B. Merriam and E. J. Tisdell, Qualitative Research: A Guide to Design and Implementation, 4th ed. San Francisco, CA: Jossey-Bass, 2016

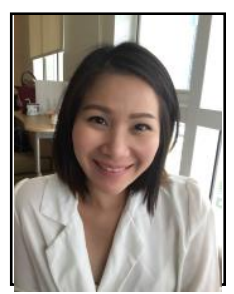

Kleddao Satcharoen was born in May 1978 Thailand. She earned her DPhil in computing (2017) and the MSc in computing (2003) from the University of Buckingham, UK, and a MA in political science (2005) from Bangkok, Thailand. She also holds BSc in management technology (2000) from King Mongkut's Institute of Technology Ladkrabang, Bangkok, Thailand.

Since 2004, she has been working as the lecturer in Faculty of Engineer, Department of Computer Engineering at King Mongkut's Institute of Technology Ladkrabang, Bangkok, Thailand. She currently teaches 3 main courses: human computer interaction, object oriented analysis and design, and web technology. Therefore, her interest is on human computer interaction area, specific in icon characteristics, perception and algorithmic/programmatic techniques for icon characteristics measurement 